\title{
Osteomyelitis on the Mandibular Malunion and Nonunion Site: A Case Report
}

\author{
Chi-Woong Song, Hyun-Joong Yoon, Sang-Hwa Lee \\ Department of Oral and Maxillofacial Surgery, Yeouido St. Mary's Hospital, \\ The Catholic University of Korea, Seoul, Korea
}

\begin{abstract}
The proper management of mandibular fractures involves reduction, rigid fixation, and immobilization to allow bone healing. Nonunion or malunion at the fractured sites is a well-known complication of fracture when the treatments are inappropriate. We present a case of left mandibular fracture due to shrapnel during the Korean War. The patients did not receive appropriate treatment at that time, so nonunion and malunion developed. Sixty years after the accident, mandibular osteomyelitis on the fracture site developed due to dental-origin inflammation. The treatment was based on relatively conservative care, such as saucerization and administration of antibiotics. There was no complication during the short-term follow-up. We present the case with literature review.
\end{abstract}

Key Words: Malunion; Nonunion; Osteomyelitis

\section{Introduction}

Patients with maxillofacial shrapnel wounds require comprehensive treatment that includes appropriate diagnosis followed by the removal of shell fragments, disinfection of wound, reduction and rigid fixation of fracture, appropriated wound closure, and prevention of further infections. The clinical outcomes can also differ from other general trauma care ${ }^{1,2)}$. If shrapnel remains within the maxilla, appropriate imaging such as X-ray is needed to remove any impacted foreign body. Failure to remove these fragments forces patients to live with them. In most cases, the presence of these unnatural materials usually poses no evident harm to the body; in some cases, however, patients can experience intermittent acute inflammation or chronic inflammation due to long-lasting local inflammation ${ }^{3)}$. If maxillary fracture occurs due to shrapnel, appropriate treatment such as rigid fixation should also be performed. Failure to fix these fractures can result in malunion and

Corresponding Author: Sang-Hwa Lee

Department of Oral and Maxillofacial Surgery, Yeouido St. Mary's Hospital, The Catholic University of Korea, 10, 63-ro, Yeongdeungpo-gu, Seoul 150-713, Korea

TEL : +82-2-3779-2148, FAX : +82-2-769-1689, E-mail : zangdak81@naver.com

Received for publication November 6, 2013; Returned after revision November 22, 2013; Accepted for publication November 29, 2013 Copyright (c) 2013 by Korean Academy of Dental Science

(C) This is an open access article distributed under the terms of the Creative Commons Attribution Non-Commercial License (http://creativecommons.org/licenses/ by-nc/3.0) which permits unrestricted non-commercial use, distribution, and reproduction in any medium, provided the original work is properly cited. 
nonunion.

Maxillofacial osteomyelitis is usually odontogenic or traumatic in origin, involving this bony infection. It can be widespread in all anatomic fasciae, and necrosis can occur ${ }^{4)}$. Treatments of osteomyelitis at the site of mandibular fracture range from conservative care such as intermaxillary fixation and drainage of infected tissue to mandibular resection and bone graft ${ }^{5,6)}$. Currently, in Korea, there are very few cases of trauma patients with shrapnel wounds outside of the military or police hospital. As a consequence, patients suffering from malunion associated with fracture of the mandible by shrapnel due to inadequate treatment of the fracture can rarely be found. We present a case report on the treatment of osteomyelitis that developed at the mandibular nonunion and malunion site 60 years after the accident with literature review.

According to the patient's statement, the treatment was limited to the basic one such as wound care and soft tissue closure (suture) at the time mandibular fracture occurred due to shrapnel. He did not receive subsequent treatment such as removal of fragments in the maxilla and reduction of mandibular fracture. As a result, the site of mandibular fracture healed with nonunion and malunion. Sixty years later, osteomyelitis developed at this site as a result of inflammation in the mandible teeth. Conservative care that includes extraction of the affected tooth, saucerization, administration of antibiotics, and continuous disinfection brought about satisfactory outcomes. Moreover, there were no particular findings of inflammation on impacted foreign bodies. Nonetheless, they were removed through surgical procedures for the mandible. A complete case report of this patient is presented here together with a review of related literature.

\section{Case Report}

A 74-year-old male patient came to our department on July 5, 2012 to have his left mandible wisdom tooth extracted. This patient had no particular intraoral discomfort but complained of food getting stuck in this area. The panoramic imaging showed a mixed pattern of radiolucent-opaque phages with

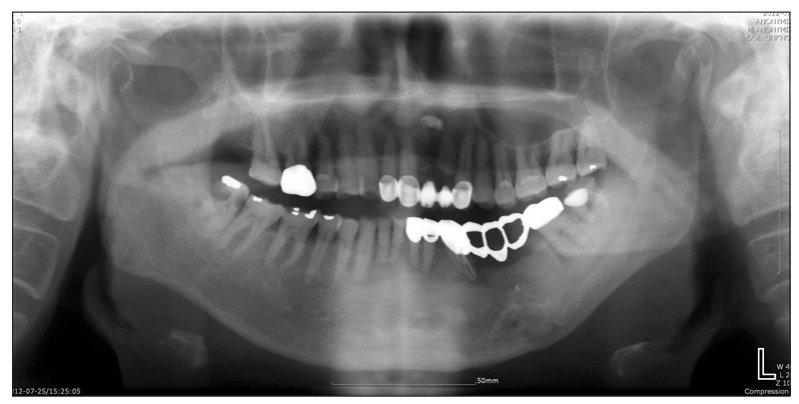

Fig. 1. Panoramic view.

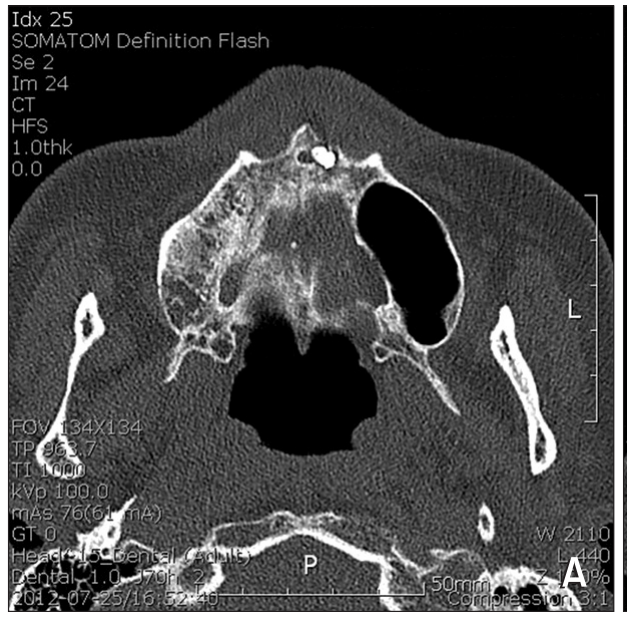

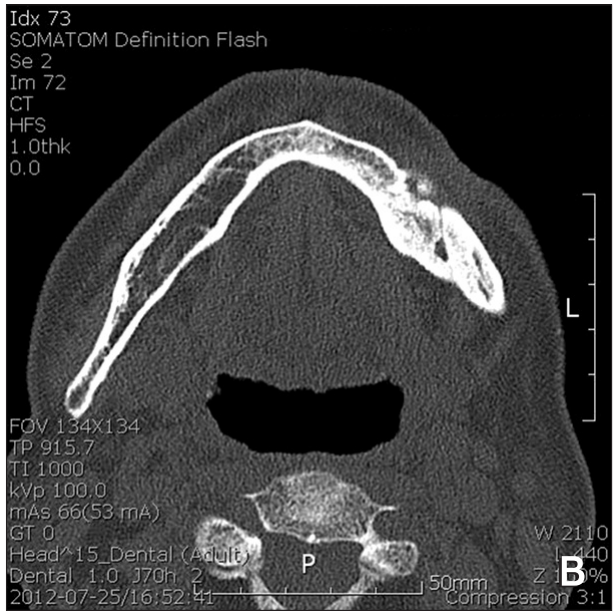

Bjo
Fig. 2. Dental computed tomography images (A: foreign body, B: nonunion and malunion site). 

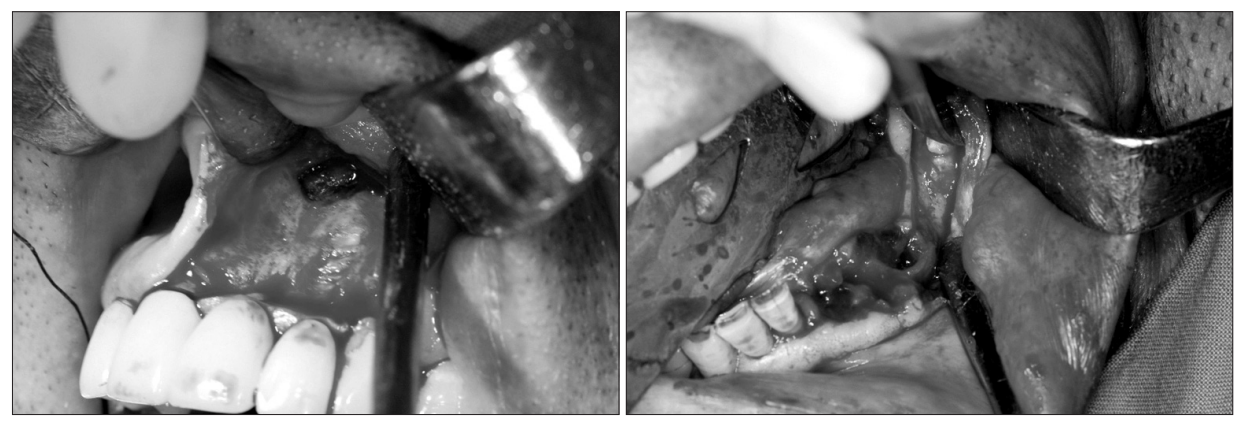

Fig. 3. Intraoral photographs.

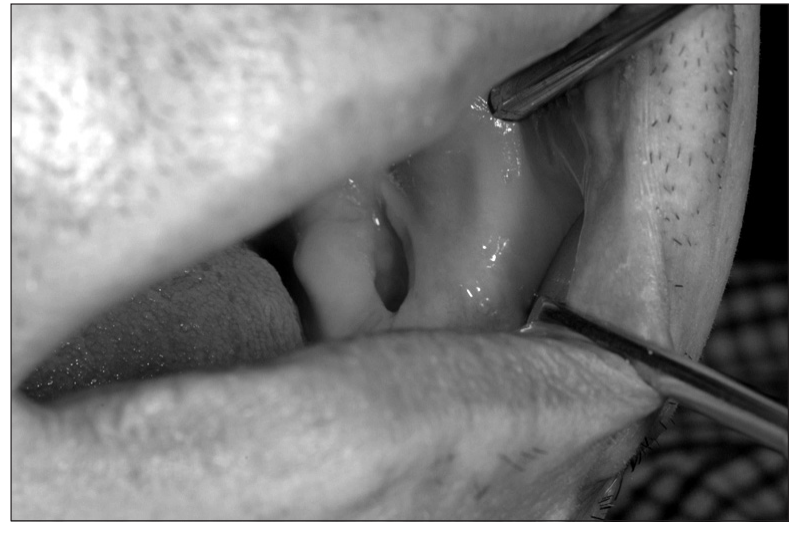

Fig. 4. Postoperative follow-up period (4 months).

unclear boundary on the inferior border of the left mandible (Fig. 1). In addition, alveolar bone loss was observed on \#31,32, 34, 37, and 38. The medical history questionnaire at the time of check showed no abnormal findings in general health care, except that he wounded his face with shrapnel during the Korean War. According to the patient's statement, he suffered mandibular fracture with loss of some upper and lower teeth due to shrapnel during the Korean War, and the treatment was limited to basic wound care and soft tissue closure; he had not received standard treatment for the hard tissue such as open reduction and fixation of the mandible and removal of foreign body in the maxilla. The computed tomography scan findings for the left mandible alveolar portion showed diffuse chronic osteomyelitis connected with the malunion and nonunion site. Moreover, radiopaque foreign body was found on the apical region of the anterior maxilla.
Note, however, that there were no specific inflammation findings around the foreign body (Fig. 2). The diagnosis was that dental-origin osteomyelitis spread to the mandibular nonunion and malunion site 60 years after the accident. Since the patient was old, and he had no specific discomfort, the most conservative treatment plan was considered. The foreign body found in the right maxillary anterior teeth was assumed to be the remaining shell fragments. The patient had no specific discomfort, and there were no inflammation findings in radiography. The surgical removal of these fragments was planned during the mandibular osteomyelitis surgery. Under general anesthesia, extraction of \#22, 31, 32, 33, 37, 38, and 48 , removal of foreign body in the maxillary anterior apical region, and saucerization were performed (Fig. 3). After the surgery, iodoforme gauze was packed on the postoperative area and changed daily during dressing. The remaining inferior border of the mandible had sufficient amount of bone even with the nonunion and malunion site, so there were no major complications such as fracture. During hospitalization, the patient was given amoxicillin/ clavulanate $1.2 \mathrm{~g}$ with IV fluid three times daily and was directed to take $625 \mathrm{mg}$ orally three times a day for one month after being discharged. Postoperative observation was performed over the next 4 months. When the patient visited for the last time, the intraoral wound was completely healed, with no pain and discomfort for the patient (Fig. 4). 


\section{Discussion}

The treatment of oral and maxillofacial wound by shrapnel requires a more comprehensive plan than other general traumas, and various complications are expected after the treatment. Trauma by shrapnel wound commonly occurs in war and conflict regions, so the treatment provided is expected to be limited ${ }^{1,2}$. The present case dealt with facial injuries due to shrapnel during the Korean War, and the patient was able to receive basic wound care only but not the proper treatment due to such special circumstances.

Shell fragments originate in the shrapnel wound and often remain in the body ${ }^{3}$. In the case of oral and maxillofacial injuries due to gunshot wounds and missile, shell fragments, maxillofacial fractures, and teeth damages commonly occur, with a high rate of occurrence ${ }^{7}$. A study surveying the penetrating injuries sustained by Israel Defense Forces soldiers during the second Palestinian uprising showed similar results. The most commonly injured body parts were the head, face, and neck $(54.2 \%)^{8}$. When this type of trauma occurs, debridement, hemostasis, soft tissue closure, open reduction of fracture, bone grafting, and complementary soft tissue repair are performed ${ }^{7}$. In the treatment of fractures, the purpose is to restore proper function through the union of fractured fragments. With improving medical technologies, fracture malunion cases due to lack of appropriate treatment are becoming less common; hence the decreasing rate of occurrence. Our patient had received basic wound care and suture only without proper care for the hard tissue such as open reduction and fixation and complete removal of foreign body due to poor underlying medical services. The left mandibular fracture was healed with nonunion and malunion, and shell fragments remained in the left maxilla without specific inflammation findings.

Maxillofacial osteomyelitis is usually odontogenic or traumatic in origin and is a mixed infection of oral bacteria involving all layers of bone where widespread necrosis occurs ${ }^{4}$. The incidence of osteomyelitis is much higher in the mandible due to the dense, poorly vascularized cortical plates and because the blood supply is primarily from the inferior alveolar neurovascular bundle. It is much less common in the maxilla due to the excellent blood supply from multiple nutrient feeder vessels. In addition, the maxillary bone is much less dense than the mandible ${ }^{9}$. The management of osteomyelitis of the maxillofacial region requires both medical and surgical intervention. Note, however, that antibiotic therapy alone is not effective in treating osteomyelitis and is usually performed together with surgical intervention ${ }^{10)}$.

This patient developed osteomyelitis at the nonunion and malunion site 60 years after the left mandibular fracture occurred, and this is a very rare case. In general, the possible causes of osteomyelitis in the mandibular fracture are complex aspects of fractures as well as oral laceration, inflammation around the teeth along the fracture line, infection through extraoral wound, and hematoma around the fractured fragments ${ }^{11}$. Osteomyelitis has been associated with multiple systemic disease including diabetes, autoimmune states, malignancies, malnutrition, and acquired immunodeficiency syndrome $^{12}$. Blood supply can also predispose the host to a bony infection ${ }^{13)}$.

In the case of this patient, nonunion and malunion of the fractured site are not thought to be the cause of osteomyelitis since the fracture occurred more than 60 years later. Rather, the cause of this osteomyelitis is highly likely to be periapical lesion of the left mandibular teeth invading this malunion area. To be more exact, nonunion and malunion of the mandible acted as a host factor to spread osteomyelitis. Since the patient is 74 years old, his low level of immunity due to his age is also deemed to be another contributing factor. This is because nonunion osseous tissues are surrounded 
by connective tissues and are consequently more susceptible to infection than normal osseous tissues. There were no particular findings of inflammation on foreign bodies in the left anterior maxilla. When foreign body such as shell fragments remains in the body, fibrous tissues cover these foreign body unless there are no immediate infections ${ }^{3)}$. In this case, however, osseous tissues surrounded shell fragments in the maxillary bone, not connective tissues. These fragments were well isolated without communicating outside, and there were no inflammation findings in the surrounding teeth. The excellent blood circulation in the maxilla-which is better than the mandible--is deemed to be another factor, causing patients to suffer no special inflammatory responses on these fragments for the past 60 years.

There are already many cases and successful treatment options introduced for the osteomyelitis of the oral and maxillofacial area. Note, however, that there are few reports showing osteomyelitis at the nonunion and malunion site due to fracture that occurred many years ago. The results of nonunion of mandibular fracture include loss of continuity of the mandible, decrease in the length of the mandible due to the absorption of fracture fragments, and fibrous pseudoarthrosis that leads to dysfunctional chewing, swallowing, speech, aesthetic, and respiratory failure ${ }^{11)}$. In this case, however, despite the nonunion and malunion findings, the continuity of the mandible was maintained, showing no significant disorder. Lost teeth due to the mandibular fracture were replaced with prosthetics; thus helping maintain the ability of chewing, swallowing, speech, and aesthetic.

In general, the causes of infection in the area of mandibular fracture are topical factors such as hematoma in the fractured area, infection of teeth along the fracture line, open lacerated wound, foreign body, lacerated wound in the periosteum, edema, lymphostasis, and unsanitary oral hygiene. Systemic factors such as age, malnutrition, and debilitating diseases can also be contributing factors ${ }^{11)}$. To treat osteomyelitis of the mandibular fracture, intermaxillary fixation should first be performed to prevent the entry of microorganism through the movement of fractured fragments. Removing teeth above the fracture line and foreign body should also be performed, and setting up the drainage areas around the osteomyelitis is one of the methods that have been proposed as conservative treatment options ${ }^{5}$. Even with these primary treatments, if nonunion persists, the removal of inflammatory fibrous tissues and scar tissues between the fractured fragments is performed along with reconstruction using bone transplantation. These methods can impose huge economic burden and pain and even cause functional and aesthetic defects to the patient. Therefore, primary conservative treatments are preferred ${ }^{6}$. This case of osteomyelitis was caused not by the mandibular fracture itself but by inflammation of dental origin penetrating along the fracture line. Such osteomyelitis was already healed with nonunion and malunion of the fractured fragments; therefore, intermaxillary fixation was not considered. The infected teeth were extracted during saucerization, and antibiotic therapy was performed. The remaining postoperative mandibular inferior border was the nonunion and malunion site, so the pathological fracture of the mandible was a concern. Nonetheless, it was healed without complications.

\section{References}

1. Zadik $Y$. The role of the military dental surgeon in treating facial injuries: a case report. Mil Med. 2007; 172: 1284-6.

2. Motamedi MH. Primary management of maxillofacial hard and soft tissue gunshot and shrapnel injuries. J Oral Maxillofac Surg. 2003; 61: 1390-8.

3. Kim SG. Treatment of foreign bodies caused by gunshot injury: 2 case reports. J Korean Assoc Oral 
Maxillofac Surg. 1996; 22: 174-88.

4. Lucchesi L, Kwok J. Long term antibiotics and calcitonin in the treatment of chronic osteomyelitis of the mandible: case report. Br J Oral Maxillofac Surg. 2008; 46: 400-2.

5. Topazian RG, Goldberg MH. Oral and maxillofacial infections. 2nd ed. Philadelphia: Saunders; 1987.

6. Kim JB, Yoo JH, Choi BH. Conservative care of nonunion owing to osteomyelitis associated with fracture of mandible: report of 3 cases. J Korean Assoc Maxillofac Plast Reconstr Surg. 2001; 23: 471-7.

7. Peled M, Leiser Y, Emodi O, Krausz A. Treatment protocol for high velocity/high energy gunshot injuries to the face. Craniomaxillofac Trauma Reconstr. 2012; 5: 31-40.

8. Lakstein D, Blumenfeld A. Israeli Army casualties in the second Palestinian uprising. Mil Med. 2005; 170: 427-30.

9. Miloro M, Ghali GE, Larsen PE, Waite P. Peterson's principles of oral and maxillofacial surgery. 2nd ed. Hamilton, London: BC Decker Publishing; 2004.

10. Lazzarini L, Lipsky BA, Mader JT. Antibiotic treatment of osteomyelitis: what have we learned from 30 years of clinical trials? Int J Infect Dis. 2005; 9: 127-38.

11. Fonseca RJ. Oral \& maxillofacial trauma. 4th ed. St. Louis: Elsevier/Saunders; 2013.

12. Marx RE. Chronic osteomyelitis of the jaws. Oral Maxillofac Clin North Am. 1991; 3: 367-81.

13. Wannfors K, Gazelius B. Blood flow in jaw bones affected by chronic osteomyelitis. Br J Oral Maxillofac Surg. 1991; 29: 147-53. 